\title{
Innovations in Storage Technology and Postharvest Science
}

\author{
P. Tonutti ${ }^{\mathrm{a}}$ \\ Scuola Superiore Sant'Anna \\ Piazza Martiri della Libertà 33 \\ 56127 Pisa \\ Italy
}

Keywords: abiotic stress, chilling injury, dynamic controlled atmosphere, integrated
postharvest protocols, metabolic profiling, proteomics, storage disorders,
transcriptomics

\begin{abstract}
Novel or improved postharvest technologies have been developed in the last 10-15 years. Some of them (e.g., 1-MCP) are already being used in several storage facilities and commercial applications, others (e.g., DCA) are just targeted for specific horticultural crops, some others are still at a preliminary or pilot-scale level and currently under evaluation. The integration of different treatments and the optimization of already existing protocols, a trend observed for the storage of several commodities, need to be assessed and validated through the combination of basic and applied research with the aim of elucidating metabolic processes and regulatory mechanisms affected by the imposed postharvest conditions. The development of "omics" technologies and the increasing number of horticultural crop genomes that have been sequenced and annotated are facilitating this new approach in postharvest science. Information and descriptions of storage-related processes at the level of transcripts, proteins and metabolites are starting to be available concerning the responses to low temperature storage including the different aptitudes to develop chilling injuries. Similarly, the biological basis of the effects of hypoxic conditions on the maintenance of quality and the onset of the related physiological disorders (superficial scald, browning) are going to be elucidated by means of integrated and systemic approaches.
\end{abstract}

\section{INTRODUCTION}

In recent years several improvements have been made in storage technologies and the postharvest manipulations of horticultural produce in order to reduce losses, maintain taste and nutritional properties, and enhance market value. Besides these main goals, innovations are also aimed at increasing the sustainability of postharvest technologies. This can be accomplished through a more rational use of energy and refrigeration, the recycling of water, the application of non-destructive technologies for quality evaluation, the reduction of chemicals, as well as by decreasing the amount of standard packaging and the introduction of environmentally-friendly or recyclable materials. Many chemicals formally used in postharvest have been (or are going to be) withdrawn and methods are being developed for hurdle technologies using a combination of physical treatments and GRAS (generally recognized as safe) compounds. In the area of produce quality, many packinghouses have facilities and equipments for quality assessment, where samples of the produce entering the packinghouse as well as those taken from packing lines are examined for quality. An important innovation is the development of technologies that can determine various aspects of quality non-destructively. In many packinghouses, fruit are automatically graded for color and blemishes by online cameras that photograph the fruit and send them to the proper sorting line. This is combined with sorting for weight and size by automatic weighing cups. Newer technologies include near-infrared spectrometers that can examine internal quality, particularly soluble solids or sugars, as well as acoustic and vibrational instruments that can measure firmness. These are

a pietro.tonutti@sssup.it 
installed on some packinghouse lines, and, in addition, hand-held instruments are being developed for use in orchards to determine optimum picking date. With an increasing awareness of safety consideration and ecological constraints, a number of different packaging concepts are being developed for fresh fruit and vegetables.

Although novel or improved technologies/devices are already being used in storage facilities and commercial applications, others are still at a preliminary or pilotscale level and currently under evaluation. In this context, conjugating and integrating basic and applied research represents the key element for successfully assessing the efficacy of these new solutions through the elucidation of metabolic processes and regulatory mechanisms affected by the imposed postharvest conditions that represent a stress experience for the produce.

The main goal of postharvest biology is precisely that of describing and understanding the relationship existing between storage conditions and components of metabolism. Given that most postharvest phenotypes are genetic traits associated with one or more genes, understanding the genetic determinants that confer quality traits in fruit and other commodities is crucial for the development of new postharvest technologies. For years, researchers have studied one gene at a time, in isolation from the wider context of other genes. A number of investigations have been done on specific structural and regulatory genes involved in quality-related metabolic pathways in different horticultural produce. As the molecular mechanisms of phenotypes and the biological basis of quality are complex, new methods of analyzing genes and their products "en masse" offer a wider view of biological events and allows study of the network through which genes, proteins and metabolites are related and communicate. The recent development of high-throughput techniques and new biotechnological approaches covering a broad field of disciplines (chemistry, physics, biology, physiology, computer science, robotics) has opened up the "omics" era in plant research, including the field of postharvest (Tonutti and Bonghi, 2009), and this is also facilitated thanks to the increasing number of horticultural crop genomes that have been sequenced and annotated.

Several technologies have emerged and are now available for measuring transcript abundance in parallel fashion, and for describing expression profiling in a particular sample (cell or tissue). An analysis of the transcriptome is a representation of all of the genes expressed, and this approach is known as transcriptomics. Besides RNA, targets of functional genomics studies are also proteins (proteomics) and metabolites (metabolomics). Functional genomics analyses are highly complementary in determining gene functioning: if transcriptional profiling describes gene expression patterns and gene regulatory networks, proteomics provides qualitative and quantitative information about proteins, and metabolomics is aimed at profiling the range of metabolites present in a sample at a given time, or under certain conditions. This multidisciplinary approach is a prerequisite for the development of Systems Biology that represents the future challenge in advanced postharvest science (Hertog et al., 2011). Up to now, most of the "omics" approaches to study the responses of horticultural produce to storage conditions have been addressed to describe gene expression profiling and only few papers have been published on proteomics and even less on metabolomics.

\section{TRENDS IN POSTHARVEST TECHNOLOGY AND NEW RESEARCH APPROACHES}

There is no doubt that the commercial application of 1-MCP on a wide range of horticultural produce can be considered one of the most important innovations in postharvest technology of the last 15 years. The impact of 1-MCP on postharvest science and technology is double: 1) it provides the potential to maintain fruit and vegetable quality after harvest, and 2) it represents a powerful tool to gain insight into the fundamental processes that are involved in ripening and senescence (Valero and Serrano, 2010). An excellent review on the relevance of 1-MCP in postharvest horticulture research has been published by Schotsmans et al. (2010) and some papers have reported the effects on transcript profiling of treatment with 1-MCP applied in combination or not 
with the traditional storage protocols and in relation to the evolution of quality parameters/physiological disorders. These studies, based on different technical approach ranging from differential screening of cDNA libraries to mRNA differential display, from microarray hybridizations to RNA sequencing and applied on different climacteric fruit such as banana (Gupta et al., 2006), peach (Ziliotto et al., 2008), apple (Costa et al., 2010), tomato (Tiwari et al., 2011), pointed out common and divergent molecular mechanisms at gene expression level possibly responsible for the different responses to $1-\mathrm{MCP}$ treatments. By using a next generation sequencing method (Solexa platform), Zuo et al. (2012) recently identified specific microRNA (miRNA), an ubiquitous class of short RNAs, that play vital roles by mediating gene silencing at post-transcriptional level. Some of the identified miRNA resulted to be differentially expressed in tomato fruit following 1-MCP treatment. These findings clearly demonstate the need of addressing the postharvest research efforts towards a holistic approach for the elucidation of the complex responses of fruit to ethylene and ethylene antagonists.

Advancements in our knowledge of the responses of different crops to postharvest conditions coupled with the stringent need of setting up more efficient, targeted, and sustainable strorage protocols are stimulating the development of innovative strategies that, for some crops, are now applied at commercial scale.

\section{Integration of Storage Protocols}

The exploitation of the synergic effects of different conditions/treatments (in combination or as sequential application) is not a novelty in postharvest. The most representative example of such approach is probably the application of controlled atmosphere (CA) in addition to optimal refrigeration. The combination of both low temperature and low $\mathrm{O}_{2}$ and high $\mathrm{CO}_{2}$ concentrations is highly effective in slowing down general metabolism, reducing ethylene production and respiration rate, thereby delaying fruit quality degradation and, in general, preventing the appearance of some physiological disorders. After the introduction of 1-MCP at commercial level, several trials have been carried out not only to identify the best concentrations in relation to the different species and cultivars, but, in particular for apples, to set up the most efficient protocols by integrating the treatment with 1-MCP with the other traditional storage parameters. The combination and interplay of different factors such as refrigeration, low oxygen/CA, and 1-MCP have been tested on apples and pears for maintaining quality (mainly, although not exclusively, firmness) and reduce the incidence of the physiological disorder superficial scald. The identification of new strategies to control the storage scald is of great importance in European countries (and those that export to EU) considering that antioxidant chemicals such as diphenylamine (DPA) and ethoxyquin have been banned by the European Commission (Calvo and Kupferman, 2012). Although results may vary depending on the cultivar and the ripening stage at harvest, combining ultra-low oxygen ( $1 \mathrm{kPa} \mathrm{O}_{2}, \mathrm{ULO}$ ) with $1-\mathrm{MCP}$ is more effective in maintaining firmness than ULO treatment alone after storage and 7 days of shelf life after storage of 'Golden Delicious' apples, and, interestingly, this effect is present also at higher storage temperature $(2.5$ instead of $1.3^{\circ} \mathrm{C}$ ) (Zanella et al., 2008). The same authors pointed out that ULO+1-MCP has an effect comparable to that of a DPA treatment in reducing the incidence of superficial scald in 'Granny Smith' apples after 6 month of refrigerated $\left(1.3^{\circ} \mathrm{C}\right)$ storage followed by $7 \mathrm{~d}$ at $20^{\circ} \mathrm{C}$. These findings (together with data published by several other authors) clearly indicate the existence of complex regulatory mechanisms that are differentially and/or synergistically affected by the imposed conditions. Studies on transcriptomic dynamics during apple fruit ripening and postharvest allowed to identify several sets of genes including transcription factors (TF) whose expression is affected by 1-MCP (Costa et al., 2010). In particular, unigenes belonging to the bZIP group and WIZZ appear to be down regulated by 1-MCP treatment, while other elements including MADS-box GDEF1, MYB TMH27 and AP2 genes are stimulated by this treatment, suggesting negative regulation by ethylene in a subset of putative ripening regulators. Considering the combination of different treatments (1-MCP, temperature, low oxygen) 
on firmness evolution and the incidence of superficial scald in stored apples, it would be of great interest to evaluate if and how the identified ethylene-dependent TF are also affected by the other imposed conditions, in particular comparing apple cultivars showing different and contrasting storage responses. The increasing amount of information and data concerning the metabolic changes occurring in apple fruit treated with 1-MCP (Lee et al., 2012) and in relation to the development of superficial scald (Rudell et al., 2009) will greatly help to complement the transcriptomic data for a more comprehensive elucidation of the processes occurring in apples stored under integrated protocols.

Considering another category of integrated storage protocols, it is recognized that pre-storage treatments with high temperature can be effective for enhancing fruit chilling tolerance during subsequent cold storage. Heat pretreatments have been shown to be effective in reducing chilling injury $(\mathrm{CI})$ in several fruit and some molecular events in response to such integrated treatments have been described. TF such as WRKY and TFIIB, together with genes encoding proteins involved in secondary metabolism, cell wall modification, oxidative damage and other stress-responsive proteins seem to be involved in the molecular mechanisms operating during heat-induced chilling tolerance in citrus fruit (Sanchez-Ballesta et al., 2003). Chilling-responsive and heat- and conditioninginduced genes have been identified in grapefruit where it seems that the heat pretreatment exerts a priming effect enabling the fruit to activate defence responses after subsequent exposure to chilling (Sapitnitskaya et al., 2006). The hot water treatment activates mainly the expression of various stress-related genes (HSP, USP, SOD) whereas the conditioning treatment activates mainly the expression of lipid membrane modification enzymes such as FAD2 and LTP.

A genome-wide transcriptional profiling analysis of RNA isolated from grapefruit flavedo using the Affymetrix Citrus GeneChip microarray revealed that exposure to chilling of grapefruit, led to downregulation of genes involved in general cellular metabolic activity, including cell wall, pathogen defence, photosynthesis, respiration, and protein, nucleic acid and secondary metabolism. On the other hand, chilling enhanced adaptation processes that involve changes in the expression of transcripts related to membranes, lipid, sterol and carbohydrate metabolism, stress stimuli, hormone biosynthesis, and modifications in DNA binding and transcription factors (Maul et al., 2008).

Information concerning the molecular responses of fruit to low temperature is essential in order to set up new protocols aimed at a more rational use of energy by modulating temperature during storage and prevent the onset of chilling injury and low temperature-associated disorders. Mechanisms that maintain citrus fruit quality during lengthy low temperature storage have been recently studied by means of a comparative transcriptomics and proteomics analysis based on digital gene expression (DGE) profiling and two-dimensional gel electrophoresis (2-DE), respectively (Yun et al., 2012). This integrated approach confirmed that low temperature up-regulates stress-responsive (such as HSP) genes and and inhibited primary metabolism, secondary metabolism and the transportation of metabolites. Calcineurin B-like protein (CBL)-CBL-interacting protein kinase complexes might be involved in the signal transduction of low temperature stress, and fruit quality is likely to be regulated by sugar-mediated auxin and abscisic acid (ABA) signalling.

Considering chilling injury and the molecular mechanisms related to the onset and appearance of CI symptoms, peach is probably one of the best characterized fruit thanks to several studies carried out based on different "omics" techniques. A cDNA microarray, named ChillPeach comprising 4261 unigenes, was obtained from harvested, ripened and stored fruit of two peach progenies contrasting for chilling injury sensitivity (Ogundiwin et al., 2008) and has been used to start identifying genes putatively involved in the appearance of the CI-related disorder named woollines. Based on a macroarray approach, Gonzales-Aguero et al. (2008), identified specific genes (cobra, endopolygalacturonase, cinnamoyl-CoAreductase) that, on the basis of the expression pattern, appeared strictly related with the development of the storage disorder. This study also indicates that 
molecular changes during fruit woolliness involve changes in the expression of genes associated with cell wall metabolism and endomembrane trafficking. Additional transcriptomic studies (analysis of EST sequences, Vizoso et al., 2009) pointed out that woolly fruits lack the increased boost of metabolic processes necessary for ripening, suggesting that the mitochondria and plastids play a major role in these processes. The isolation of cold-induced peach genes revealed that the promoter sequence of these genes contain several cis-regulatory elements such as DRE/CRT, ABRE, MYCR (MYC recognition site) and MYBR (MYB recognition site) that are related to stress response, specifically to cold/dehydration (Tittarelli et al., 2009). Besides differences in transcript accumulation, wolly and juicy fruit resulted to be also different when a proteomics approach was used. Accumulations of proteins such as endopolygalacturonase, catalase, NADP-dependent isocitrate dehydrogenase, pectin methylesterase and dehydrins were found to be different in healthy and CI fruit suggesting that 'response to stress', 'cellular homeostasis', 'metabolism of carbohydrates' and 'amino acid metabolism' are major biological processes involved in the development of low temperature storage disorders (Nilo et al., 2010).

\section{Optimization of Postharvest Strategies}

If we look at the evolution of the CA technology since its early practical applications for apple storage, one trend is evident: a steady decrease of oxygen and increase of $\mathrm{CO}_{2}$ concentrations used in the storage rooms. The standard technology, based on an oxygen concentration of about $2-3 \mathrm{kPa}$, has, in the last 15 years, markedly changed and innovations are represented by CA-based methods characterized in particular by a reduction of $\mathrm{O}_{2}$ levels. One example is represented by the ultra-low oxygen (ULO) technology where $\mathrm{O}_{2}$ is maintained near $1 \mathrm{kPa}$, and ILOS (Initial Low $\mathrm{O}_{2}$ Stress) in which $\mathrm{O}_{2}$ levels are maintained as low as $0.25-0.7 \mathrm{kPa}$ for short time periods after harvest. A further step, thanks to the advances in technology that allow sensing of fruit responses to stress hypoxic stress conditions, is represented by the dynamic CA (DCA). With this technology, fruit are kept at much lower $\mathrm{O}_{2}$ concentrations than the "safe" - but not optimal - levels, but this concentration is promptly adjusted in relation to the fruit metabolic responses. The main parameters used to monitor the metabolic responses are based on the measurement of ethanol production by the fruit and/or the chlorophyll fluorescence (Schouten et al., 1997; Prange et al., 2002, 2003). The advantages of DCA are that this is a chemical-free technology, provide excellent results for long term storage and can be effective in controlling the superficial scald with no need of using antioxidant chemicals. One disadvantage is that it requires highly airtight rooms and electronic atmosphere control.

The reduction of oxygen levels at concentrations as low as $0.4 \mathrm{kPa}$ for periods longer than few days represents a high-level stress condition and has a profound impact on the fruit metabolism. The optimization of this technique (also for the possible storage of other crops than apples and pears) must be based on the elucidation of the hypoxiainduced/affected processes, thus reducing the risks of the appearance of low-oxygenassociated storage disorders and the decrease of quality parameters (off-flavours, tissue browning, etc.). As observed in model plant species, lack of oxygen causes a reduction in respiratory efficiency and, as a consequence, in energy production (Gupta et al., 2009). Therefore, under hypoxia/anoxia ATP synthesis is mostly provided by glycolysis coupled with NAD regenerative pathways, including ethanolic fermentation and alanine production (Ricoult et al., 2006; Ismond et al., 2003). These processes occur also in fruit under altered atmosphere composition and it can be hypothesized that metabolic imbalance may lead to the development of low-oxygen induced physiological disorders. Preliminary metabolomics and proteomics studies have been performed on pears stored under low oxygen conditions and in relation to the development of the core breakdown disorder. Indeed, the metabolic profiling of brown and sound tissue using GC-EI-TOFMS, showed that brown tissue was clearly characterized by a decrease of malic acid and an increase in fumaric acid and gamma aminobutyric acid (GABA), which indicated a 
reduced metabolic activity at the level of the Krebs cycle and a putative block of the GABA shunt pathway (Pedreschi et al., 2009a). Interestingly, GABA and gluconic acid have been shown to be metabolic markers for core breakdown. When stored under anoxia, pear fruit show, by means of a differential gel electrophoresis (DIGE) approach, marked changes in proteins involved in respiration, protein synthesis, and defense mechanisms, including some PR proteins (Pedreschi et al., 2009b). It will be interesting to assess whether and how these metabolic and proteomic profiles change according to different oxygen concentrations such as those used in DCA. Surprisingly, and differently from model species such as Arabidopsis and rice that have been studied in relation to hypoxia/anoxia occurring during flooding, no data concerning transcript profiling in stored fruit under low oxygen are so far available. In a preliminary experiment, 'Granny Smith' apples stored for 22 days at 0.4 and $0.8 \mathrm{kPa}$ oxygen concentrations have been analyzed in terms of transcript profiling by means of RNAseq (Tonutti, Ruperti, Velasco, unpublished). When compared with fruit at harvest, almost 14,000 genes resulted to be transcribed in total: most of them resulted affected under both conditions, but about 1,400 were selectively regulated in $0.4 \mathrm{kPa}$ sample and other 1,400 in the $0.8 \mathrm{kPa}$ sample. This would indicate that this apparently slight difference in terms of oxygen concentration has a marked effect in regulating gene expression. This is true when analyzing specific genes such as alcohol dehydrogenases (ADH), that show significant difference between samples in terms of transcript accumulation (marked increase in $0.4 \mathrm{kPa}$, less pronounced increase in $0.8 \mathrm{kPa}$ ). The different expression of specific Ethylene Responsive Factors (ERF), in particular those belonging to the group VII, that have been shown to be involved in lowoxygen signalling in plants through the N-end rule pathway (Gibbs et al., 2011; Licausi et al., 2011, 2010), would indicate a possible role of these TF in the oxygen sensing mechanisms present also in fruit tissues. This would open interesting perspectives in terms of comparative analyses of different apple cultivars (but also other horticultural species) showing different sensitivity and metabolic reactivity (e.g., variable level of ethanol) to low oxygen conditions during storage.

\section{Literature Cited}

Calvo, G. and Kupferman, E. 2012. Current DPA and ethoxyquin situation and alternatives to superficial scald control in apples and pears. Acta Hort. 945:51-54.

Costa, F., Alba, R., Schouten, H., Soglio, V., Gianfranceschi, L., Serra, S., Musacchi, S., Sansavini, S., Costa, G., Fei, Z. and Giovannoni, J. 2010. Use of homologous and heterologous gene expression profiling tools to characterize transcription dynamics during apple fruit maturation and ripening. BMC Plant Biology 10:229.

Gibbs, D.J.D., Lee, S.C.S., Isa, N.M.N., Gramuglia, S.S., Fukao, T.T., Bassel, G.W.G., Correia, C.S.C. et al. 2011. Homeostatic response to hypoxia is regulated by the Nend rule pathway in plants. Nature 479:415-418.

Gonzalez-Aguero, M., Pavez, L., Ibanez, F., Pacheco, I., Campos-Vargas, R., Meisel, L.A., Orellana, A., Retamales, J., Silva, H. and Gonzalez, M. 2008. Identification of woolliness response genes in peach fruit after post-harvest treatments. J. Exp. Bot. 59:1973-1986.

Gupta, S.M., Srivastava, S., Sane, A.P. and Nath, P. 2006. Differential expression of genes during banana fruit development, ripening and 1-MCP treatment: presence of distinct fruit specific, ethylene induced and ethylene repressed expression. Postharvest Biology and Technology 42:16-22.

Gupta, K.J., Zabalza, A. and van Dongen, J.T. 2009. Regulation of respiration when the oxygen availability changes. Physiol. Plant. 137:383-391.

Hertog, M., Rudell, D.R., Pedreschi, R., Schaffer, R.J., Geeraerd, A.H., Nicolaï, B. and Ferguson, I. 2011. Where systems biology meets postharvest. Postharvest Biol. Technol. 62:223-237.

Ismond, K.P., Dolferus, R., De Pauw, M., Dennis, E.S. and Good, A.G. 2003. Enhanced low oxygen survival in Arabidopsis through increased metabolic flux in the fermentative pathway. Plant Physiol. 132:1292-1302. 
Lee, J., Rudell, D., Davies, P. and Watkins, C. 2012. Metabolic changes in 1methylcycloprene (1-MCP)-treated 'Empire' apple fruit during storage. Metabolomics 8:742-753.

Licausi, F., Kosmacz, M., Weits, D.A., Giuntoli, B., Giorgi, F.M., Voesenek, L.A.C.J. and Perata, P. 2011. Oxygen sensing in plants is mediated by an $\mathrm{N}$-end rule pathway for protein destabilization. Nature 479:419-422.

Licausi, F., van Dongen, J.T., Giuntoli, B., Novi, G., Santaniello, A., Geigenberger, P. and Perata, P. 2010. HRE1 and HRE2, two hypoxia-inducible ethylene response factors, affect anaerobic responses in Arabidopsis thaliana. The Plant Journal 62:302315.

Maul, P., McCollum, G.T., Popp, M., Guy, C.L. and Porat, R. 2008. Transcriptome profiling of grapefruit flavedo following exposure to low temperature and conditioning treatments uncovers principal molecular components involved in chilling tolerance and susceptibility. Plant Cell Environ. 31:752-768.

Nilo, R., Saffie, C., Lilley, K., Baeza-Yates, R., Cambiazo, V., Campos-Vargas, R. and Gonzalez, M. 2010. Proteomic analysis of peach fruit mesocarp softening and chilling injury using difference gel electrophoresis (DIGE). BMC Genomics 11(1):43.

Ogundiwin, E.A., Marti, C., Forment, J., Pons, C., Granell, A., Gradziel, T.M., Peace, C.P. and Crisosto, C. 2008. Development of ChillPeach genomic tools and identification of cold responsive genes in peach fruit. Plant Mol. Biol. 68:379-397.

Pedreschi, R., Franck, C., Lammertyn, J., Erban, A., Kopka, J., Hertog, M. and Verlinden, B. 2009a. Metabolic profiling of 'Conference' pears under low oxygen stress. Postharvest Biol. Technol. 51:123-130.

Pedreschi, R., Hertog, M., Robben, J. and Lilley, K. 2009b. Gel-based proteomics approach to the study of metabolic changes in pear tissue during Storage. J. Agri. Food Chem. 57:6997-7004.

Prange, R., DeLong, J., Harrison, P., Leyte, J. and McLean, S.D. 2003. Oxygen concentration affects chlorophyll fluorescence in chlorophyll-containing fruit and vegetables. J. Amer. Soc. Hort. Sci. 128:603-607.

Prange, R., DeLong, J., Leyte, J. and Harrison, P. 2002. Oxygen concentration affects chlorophyll fluorescence in chlorophyll-containing fruit. Postharvest Biol. Technol. 24:201-205.

Ricoult, C., Orcaray Echeverria, L., Cliquet, J.B. and Limami, A.M. 2006. Characterization of alanine aminotransferase (AlaAT) multigene family and hypoxic response in young seedlings of the model legume Medicago truncatula. J. Exp. Bot. 57:3079-3089.

Rudell, D., Mattheis, J. and Hertog, M. 2009. Metabolomic change preceedes apple superficial scald symptoms. J. Hort. Sci. Biotech. 75:271-276.

Sanchez-Ballesta, M.T., Lluch, Y., Gosalbes, M.J., Zacarias, L., Granell, A. and Lafuente M.T. 2003. A survey of genes differentially expressed during long-term heat-induced chilling tolerance in citrus fruit. Planta 218:65-70.

Sapitnitskaya, M., Maul, P., McCollum, G.T., Guy, C.L., Weiss, B. and Samach, A. 2006. Postharvest heat and conditioning treatments activate different molecular responses and reduce chilling injuries in grapefruit. J. Exp. Bot. 57:2943-2953.

Schotsmans, W., Prange, R. and Binde, B. 2010. 1-Methylcyclopropene: mode of action and relevance in postharvest horticulture research. Horticultural Reviews 35:263-313.

Schouten, S.P., Prange, R., Verschoor, J.A., Lammers, T.R. and Oosterhaven, J. 1997. Improvement of quality of 'Elstar' apples by dynamic control of ULO conditions. Proc. $7^{\text {th }}$ Intl. Controlled Atmosphere Res. Conf. Davis, Calif. 2:71-78.

Tittarelli, A., Santiago, M., Morales, A., Meisel, L.A. and Silva, H. 2009. Isolation and functional characterization of cold-regulated promoters, by digitally identifying peach fruit cold-induced genes from a large EST dataset. BMC Plant Biology 9:121.

Tiwari, K. and Paliyath, G. 2011. Microarray analysis of ripening-regulated gene expression and its modulation by $1-\mathrm{MCP}$ and hexanal. Plant Physiol. Biochem. 49:329-340. 
Tonutti, P. and Bonghi, C. 2009. Advanced technologies and integrated approaches to investigate the molecular basis of fresh produce quality. p.561-582. In: W.W.J. Florkowski, R.L. Shewfelt, B. Brueckner and S.E. Prussia (eds.), Postharvest Handling: A Systems Approach, $2^{\text {nd }}$ Ed. Academic Press.

Valero, D. and Serrano, M. 2010. Postharvest Biology and Technology for Preserving Fruit Quality. CRC Press, Boca Raton, FL.

Vizoso, P., Meisel, L.A., Tittarelli, A., Latorre, M., Saba, J., Caroca, R. and Maldonado, J. 2009. Comparative EST transcript profiling of peach fruits under different postharvest conditions reveals candidate genes associated with peach fruit quality. BMC Genomics 10:423.

Yun, Z., Jin, S., Ding, Y., Wang, Z., Gao, H., Pan, Z. and Xu, J. 2012. Comparative transcriptomics and proteomics analysis of citrus fruit, to improve understanding of the effect of low temperature on maintaining fruit quality during lengthy post-harvest storage. Journal of Experimental Botany 63:2873-2893.

Zanella, A., Cazzanelli, P. and Rossi, O. 2008. Dynamic controlled atmosphere (DCA) storage by the means of chlorophyll fluorescence response for firmness retention in apple. Acta Hort. 796:77-82.

Ziliotto, F., Begheldo, M., Rasori, A., Bonghi, C. and Tonutti, P. 2008. Transcriptome profiling of ripening nectarine (Prunus persica L. Batsch) fruit treated with 1-MCP. J. Exp. Bot. 59:2781-2791.

Zuo, J., Zhu, B., Fu, D., Zhu, Y., Ma, Y., Chi, L., Ju, Z., Wang, Y., Zhai, B. and Luo, Y. 2012. Sculpting the maturation, softening and ethylene pathway: the influences of microRNAs on tomato fruits. BMC Genomics 13:7. 